\title{
Adolescentes urbanos: conocimientos sobre la atención de salud sexual y reproductiva
}

\author{
M. XIMENA LUENGO CHARATH ${ }^{1}$, TERESA MILLÁN KLÜSSE ${ }^{2}$, \\ ANA J. ZEPEDA ORTEGA³, MARCIA TIJERO MÉNDEZ ${ }^{4}$ \\ 1. Médico-Cirujano, especialista en Adolescencia, Magíster en Salud Pública. Instituto Chileno de Medicina \\ Reproductiva-ICMER; Facultad de Medicina, Universidad de Chile. \\ 2. Matrona, Magíster en Salud Pública. Departamento de Pediatría y Cirugía Infantil Occidente, Facultad de Medicina \\ Occidente, Universidad de Chile. \\ 3. Magíster en Bioestadística. Instituto Chileno de Medicina Reproductiva-ICMER \\ 4. Socióloga, Magíster en Género. Instituto Chileno de Medicina Reproductiva-ICMER.
}

\begin{abstract}
Urban adolescents: knowledge about sexual and reproductive health care

Objective: To identify the knowledge about sexual and reproductive health (SRH) care among adolescent participants of the public health care system. Subjects and Methods: Stratified sample of 368 adolescents from low-income and high teenager's pregnancy rate communities in Santiago, Chile. A self-administered, anonymous and confidential survey asked about knowledge, use of services and friendly care characteristics. Results: $52.9 \%$ of respondents knew of SRH care and from these, $22.1 \%$ had used it. Predictors for service use were: being female, been dating a boyfriend/girlfriend, having received sex education at home and not living with parents. Most participants positively evaluated the SRH service. Reasons for not seeking SHR care were: no need it, embarrassment to consult and lack of information on this type of care availability. Conclusions: Low percentage of school adolescents demand SRH attention. The care provided in the public system is well evaluated. Teens who demand professional attention to solve SHR needs are: women, who are in a relationship and those who have received sex education at home. Furthermore, no need for care, embarrasment and lack of information are factors that affect the consultation.
\end{abstract}

(Key words: Adolescents, sexual and reproductive health, public health system).

Rev Chil Pediatr 2012; 83 (6): 540-551

\section{RESUMEN}

Objetivo: Identificar entre adolescentes estudiantes beneficiarios del sistema público de salud, conocimientos sobre la atención de salud sexual y reproductiva (SSR). Paciente y Método: Muestra estratificada, 368 adolescentes de comunas de bajos ingresos y elevados nacimientos en adolescentes, en Santiago-Chile. Encuesta

Recibido el 28 de octubre de 2011, devuelto para corregir el 30 de enero de 2012, segunda versión el 13 de marzo de 2012 , aceptado para publicación el 5 de mayo de 2012.

Esta investigación fue realizada con el apoyo financiero del Special Programme of Research, Development and Research Training in Human Reproduction, World Health Organization (Project A55078).

Este trabajo cumple con los requisitos sobre consentimiento /asentimiento informado, comité de ética, financiamiento, estudios animales y sobre la ausencia de conflictos de intereses según corresponda.

Correspondencia a:

Dra. Ximena Luengo Charath

E-mail: ximeluengo@vtr.net 
autoaplicada, anónima y confidencial, preguntó conocimientos, uso de servicios y características de la atención amigable. Resultados: $52,9 \%$ de los encuestados sabía de la atención de SSR y, de ellos, 22,1\% había consultado. Variables predictivas para utilización de los servicios fueron: ser mujer, haber estado saliendo con un novio/a, haber recibido educación sexual en la familia y no vivir con los padres. La mayoría de los consultantes evaluó positivamente la atención de SSR. Razones porque no solicitaron atención de SSR fueron: no tener necesidad, vergüenza de consultar y falta de información sobre disponibilidad de este tipo de atención. Conclusiones: Escaso porcentaje de adolescentes escolares demanda atención de SSR. La atención recibida en el sistema público es bien evaluada. Adolescentes que demandan atención profesional para resolver necesidades de SSR son: mujeres, aquellos que están en relación de pareja y los que han recibido educación sexual en la familia. No necesitarla, vergüenza y falta de información, son factores que afectan la consulta.

(Palabras clave: adolescentes, salud sexual y reproductiva, sistema público de salud).

Rev Chil Pediatr 2012; 83 (6): 540-551

\section{Introducción}

Los adolescentes, por largo tiempo, han sido considerados un grupo etario con buena salud $^{1,2}$ y que acceden poco a los servicios correspondientes, probablemente en parte porque éstos no han respondido a sus requerimientos y necesidades. En general, los adolescentes dudan en buscar atención de salud por problemas psicológicos, mentales o psicosociales y, la aparente falta de problemas de salud, podría deberse a una subestimación de los mismos y a la falta de reconocimiento de la necesidad de cuidados preventivos en ellos ${ }^{3,4}$.

Las características de los Servicios de Salud Amigables para Adolescentes se han documentado ampliamente a nivel internacional ${ }^{5-10}$. En teoría, los servicios públicos chilenos de Atención Primaria reúnen varios de los criterios para ser considerados "amigables para adolescentes”, por ejemplo: son gratuitos, geográficamente cercanos y se basan en lineamientos y políticas enfocadas en los derechos de los adolescentes ${ }^{11,12}$. Se sabe que la confidencialidad es un factor importante en la atención de los adolescentes, determinante especialmente en aquellos que necesitan cuidados en salud mental y en salud sexual y reproductiva (SSR). La confidencialidad ha sido válidamente reconocida, recientemente, en varios documentos del Sistema de Salud chileno ${ }^{13-15}$. La importancia de este elemento en la consulta, radica en que es ampliamente conocido que la duda sobre el resguardo de la confidencialidad limita el acceso al cuidado de salud de los adolescentes más vulnerables y que requieren servicios, es- pecialmente de mujeres con necesidades en el área de la SSR ${ }^{16,17}$.

Informes de diversas regiones del mundo muestran que los adolescentes necesitan ayuda para prevenir embarazos no deseados y VIH/SIDA ${ }^{18-21}$. En muchos países de América Latina, la SSR es un área prioritaria ${ }^{22-24}$. Por ejemplo en Chile, reducir la proporción de nacimientos en adolescentes, en especial en las menores de 15 años, es una meta que el país ha comprometido entre los Objetivos de Desarrollo del Milenio ${ }^{25}$. Aunque los logros del Sistema de Salud Público en Chile - de acuerdo con diversos indicadores- se encuentran entre los mejores de América Latina ${ }^{26,27}$, el país experimenta en algunas áreas dificultades similares al resto de la región. Los servicios de salud adolecen de acceso igualitario y algunos indicadores específicos de SSR muestran grandes inequidades. Los adolescentes que constituyen el 18\% de la población, según algunos datos estadísticos se encuentran en desventaja y, en particular, aquellos de niveles socioeconómicos más vulnerables. Por ejemplo, para el censo 2002, el $77 \%$ de las madres adolescentes provenían de los dos quintiles de menor ingreso ${ }^{28,29}$. Según la última Encuesta Nacional de Juventud, más de tres cuartos $(76,1 \%)$ de los jóvenes de 15 a 29 años de mayores ingresos, usaron métodos anticonceptivos en su primera relación sexual, versus sólo el 39,3\% en aquellos de ingresos más bajos. Más aún, actualmente en Chile la proporción de adolescentes bajo 18 años de edad sexualmente activos en los niveles socioeconómicos altos es de 55\%, mientras en el nivel más bajo es de $69 \%{ }^{30}$. Desigualdades de este tipo 
han motivado al Ministerio de Salud a desarrollar a nivel de la Atención Primaria de Salud, servicios más amigables con los adolescentes, minimizando las barreras de acceso, aumentando la intervenciones ya existentes y mejorando la calidad de los servicios para adecuarlos a las necesidades de los y las adolescentes ${ }^{31}$.

Una encuesta nacional solicitada por el Ministerio de Educación el año 2004 y realizada con estudiantes de los seis cursos más altos de las escuelas, indicó que en general, sólo $56 \%$ de los estudiantes había recibido educación sexual en forma regular y la frecuencia de dicha actividad disminuía al aumentar la edad de los adolescentes. Los estudiantes consideraron a la madre como la persona más adecuada en la familia para educar en sexualidad $(81,6 \%)$, seguida por el padre $(59,2 \%)$ y, a los profesores y el equipo de salud, se les asignó el tercer $(49,8 \%)$ y cuarto lugar $(44,3 \%)$, respectivamente ${ }^{32}$.

En este contexto, los objetivos del presente estudio, de diseño mixto cuali-cuantitativo, fueron examinar qué conocimientos tenían estudiantes adolescentes beneficiarios del sistema público de salud, provenientes de comunas del área urbana de la Región Metropolitana de Chile, desfavorecidas en lo socioeconómico y con numerosos nacimientos en adolescentes. El foco estuvo puesto en: los conocimientos sobre la atención de salud sexual y reproductiva, identificar sus necesidades y conocer las características deseables para hacer más amigables este tipo de atención destinada a ellos. En este artículo se presentan los hallazgos del componente cuantitativo del estudio realizado.

\section{Personas y Método}

\section{Diseño y muestra}

La investigación se realizó en el año 2007, mediante un diseño cuali-cuantitativo. La dimensión cuantitativa consistió en un estudio de corte transversal y fue la primera etapa de este trabajo. La muestra fue representativa de adolescentes estudiantes de las escuelas públicas que se ubicaban en tres comunas con alta proporción de población adolescente, altos niveles de pobreza y elevado número de nacimientos de madres adolescentes, del área urbana de la Región Metropolitana. Acorde con los datos demográficos y de salud al momento del estudio, las comunas que presentaban estas características fueron: El Bosque, La Pintana y Cerro Navia. Además, se decidió acotar el estudio a colegios con alumnos de ambos sexos y para incluir adolescentes de diferentes edades, se consideraron tres niveles de educación: $8^{\circ}$ año de Enseñanza Básica, $2^{\circ}$ y $4^{\circ}$ año de Enseñanza Media.

El tamaño muestral fue estimado para muestras multi-propósitos, mediante el algoritmo propuesto por Sánchez-Crespo ${ }^{33}$. Para ello se consideró la construcción de intervalos de confianza del 95\% para los estimadores de proporción, con un error de estimación de 5\% y un efecto de diseño de 1,2. El tamaño estimado fue de 357 alumnos, el cual incluía un 20\% extra para cubrir la posible falta de respuesta. Este número se consideró adecuado también para un análisis de regresión múltiple que incluyera un máximo de 25 covariables, a un $\mathrm{R}^{2}$ de 0,35 , con un valor de probabilidad de $5 \%$ y una potencia de $80 \%{ }^{34}$.

El diseño muestral fue bietápico, estratificado por subdivisión geográfica y por niveles de educación. El marco muestral fue el Directorio de Colegios de la Región Metropolitana para el año 2006, mediante el cual se estableció que el universo en estudio era 17 colegios con un total de 5482 alumnos en los niveles educacionales definidos para la investigación. La unidad primaria de muestreo fue la escuela, estratificada por comuna, seleccionándose 6 colegios de un total de 17, con probabilidad proporcional al número de estudiantes. En la segunda etapa del muestreo, se utilizó el método del sombrero para seleccionar al azar a uno o más cursos en cada uno de los estratos educacionales, al interior de cada escuela (segunda unidad de muestreo). Todos los estudiantes de los cursos seleccionados tenían la posibilidad teórica de participar en el estudio, los que sumaban 476 alumnos. Para participar, ellos debían ser autorizados por sus padres o tutores, estar presentes al momento de la encuesta y consentir su participación en el estudio. Por tales razones, el número final de participantes fue de 368. El tamaño de la muestra logrado superó el $100 \%$ de lo calculado para este estudio. 


\section{Encuesta}

La participación fue voluntaria, anónima y confidencial, los estudiantes y sus padres fueron informados del estudio mediante una carta, recibiendo un formulario de Consentimiento Informado para padres que debía ser entregado debidamente firmado. Luego, cada estudiante firmó también el Consentimiento Informado correspondiente, previo a responder la encuesta. En los casos en que los consentimientos de los padres no fueron devueltos en un tiempo razonable, el Director del establecimiento educacional previa revisión del instrumento, autorizó por escrito según lo permite la normativa educacional chilena, la participación de sus alumnos en la encuesta, a excepción de aquellos que no dieron su consentimiento y por lo tanto, manifestaron explícitamente su deseo de no participar. El estudio fue aprobado en sus aspectos éticos, por dos Comités de Ética: uno local independiente que evalúa los proyectos de ICMER y otro de la OMS, agencia que financió el estudio. El cuestionario aplicado fue diseñado por el equipo de investigadoras para los fines del estudio, revisado por jueces expertos y luego validado en estudiantes de similares características socioeconómicas y nivel educacional, pero en una subdivisión geográfica no incluida en la muestra. Los hallazgos de este procedimiento permitieron perfeccionar el formato final del instrumento. La encuesta cubrió cinco ejes temáticos: 1) Antecedentes personales: edad, sexo, constitución de la familia y relaciones interpersonales familiares; 2) Servicios de salud en general: tipo de sistema de salud al que está adscrito/a, conocimiento del Consultorio de Atención Primaria de su barrio, lugar dónde resuelve habitualmente sus necesidades de salud, quién decide en la familia consultar o quién lo/a acompaña a consultar y, la experiencia como usuario/a de la atención primaria; 3) Atención de SSR: incluyendo el conocimiento de este tipo de atención, acceso a dichos servicios y, su experiencia como usuarios de éstos; 4) Salud sexual y reproductiva: conocimientos, relaciones de pareja, cercanía y creencias respecto del embarazo adolescente; 5) Otras características personales: amistades, uso del tiempo libre, rendimiento académico y expectativas futuras, conductas de riesgo (uso de sustancias, violencia), religión, trabajo y antecedentes de haber recibido o no educación sexual. En el módulo sobre servicios de SSR, se incluyó una pregunta sobre la importancia que le asignan a las características de los servicios amigables para adolescentes ${ }^{5}$. Esta pregunta sólo se incluyó en la encuesta de los estudiantes de Enseñanza Media $\left(2^{\circ}\right.$ y $4^{\circ}$ Medio) y consistió en un listado de características a las cuáles se le debía asignar el grado de importancia en una escala de 1 a 5 , siendo 1 lo menos importante y 5 lo más importante.

La encuesta fue aplicada por los investigadores, en la sala de clases de cada escuela, en presencia de los profesores pero sin ingerencia de ellos en el proceso. Todos los alumnos previamente autorizados y que accedieron a participar voluntariamente, respondieron el cuestionario en forma simultánea, el que una vez finalizado fue depositado por cada estudiante en una caja tipo buzón.

\section{Análisis de los datos}

La confrontación de la composición de la muestra $(\mathrm{n}=368)$ con la población estudiantil de la cual se obtuvo $(n=5482)$, hizo necesario aplicar un factor de ponderación que corrigiera la sub y sobre representación por sexo y subdivisión geográfica. Así, la muestra que se usó para los análisis reflejó de mejor forma a la población objetivo. Luego se identificaron a los alumnos cuya familia estaba cubierta por el sistema público de salud. Esto se hizo mediante dos preguntas: tipo de seguro de salud y lugar donde generalmente resolvían o consultaban por sus necesidades de salud (ambos podían ser público, privado u otro). El análisis bi y multivariado sólo incluyó a estudiantes beneficiarios de los servicios públicos, debido a que esa era la población foco del estudio.

La variable dependiente que se utilizó para el análisis multivariado, fue la declaración de haber consultado por atención de SSR en el sistema público. Las variables independientes fueron las características de los estudiantes, teniendo en cuenta la estratificación de la muestra (curso y subdivisión geográfica), así como también a las estratificaciones post-muestrales, tales como: sexo y edad. Esta última fue categorizadas en grupos: "menores de 15 años" y 
de "15 años y más" para homogenizar las experiencias y vivencias de los adolescentes, de acuerdo al período de desarrollo en que se encontraban y al corte de grupo poblacional que realizan los servicios de salud. Esto permitió la post estratificación antes mencionada y facilitó la interpretación de sus resultados.

Además, se construyeron 2 indicadores, uno de nivel de conocimiento en SSR y otro de proximidad a la actividad sexual, los cuales también fueron incorporados al análisis multivariado. El primer indicador correspondió a la suma de respuestas correctas respecto de: uso del condón y prevención de enfermedades de transmisión sexual, la fertilidad masculina, el período fértil femenino, la posibilidad que tienen las adolescentes de usar la mayoría de los métodos anticonceptivos $\mathrm{y}$, por último, si la anticoncepción oral de emergencia es o no abortiva. El puntaje obtenido fue clasificado en tres categorías (bajo, intermedio y bueno). El segundo indicador de cercanía con la actividad sexual, se obtuvo calculando la diferencia entre la edad que en opinión del o de la adolescente era la ideal para iniciar vida sexual activa y la edad cronológica actual del adolescente, diferencia que fue categorizada en "un año o menos" y "mayor a un año". Este último se consideró una medida indirecta de proximidad al inicio de la actividad sexual.

En el análisis multivariado sólo se incluyeron las variables cuya asociación con la consulta por atención de SSR en los Servicios Públicos, resultó estadísticamente significativa en el análisis bivariado. El modelo final fue evaluado de manera que cumpliera con los supuestos estadísticos. El análisis estadístico se realizó con el paquete Statistical Analysis System $(\mathrm{SAS} \otimes)^{35}$.

\section{Resultados}

Las características de los 368 estudiantes que respondieron la encuesta se muestran en la tabla 1. El 88,3\% de estos estudiantes $(n=325)$ eran beneficiarios del sistema público de salud, al cual acceden gratuitamente, y su distribución por sexo fue muy similar (51,1\% hombres/48,9\% mujeres). Del total de adolescentes beneficiarios del sistema público, el 18,5\% $(\mathrm{n}=60)$ había utilizado servicios de SSR, aunque hubo algunos estudiantes que desconocían que su consulta fue por

Tabla 1. Total de adolescentes encuestados y beneficiarios del Sistema Público de Salud, por sexo, edad, nivel de educación y subdivisión geográfica

\begin{tabular}{|c|c|c|c|c|}
\hline \multirow[t]{2}{*}{ Características } & \multicolumn{2}{|c|}{ Número total de encuestados } & \multicolumn{2}{|c|}{ Número de beneficiarios del sistema público } \\
\hline & Muestral & Ponderado & Muestral & Ponderado \\
\hline Total (n) & 368 & 368 & 325 & 325 \\
\hline \multicolumn{5}{|l|}{ Sexo } \\
\hline Hombre & 191 & 185 & 164 & 166 \\
\hline Mujer & 177 & 183 & 161 & 159 \\
\hline \multicolumn{5}{|c|}{ Nivel de educación } \\
\hline 8 Básico & 183 & 127 & 164 & 113 \\
\hline $2^{\circ}$ Medio & 125 & 143 & 108 & 124 \\
\hline $4^{\circ}$ Medio & 60 & 98 & 53 & 88 \\
\hline \multicolumn{5}{|c|}{ Área geográfica (comuna) } \\
\hline Cerro Navia & 88 & 112 & 79 & 100 \\
\hline El Bosque & 129 & 127 & 110 & 111 \\
\hline La Pintana & 151 & 129 & 136 & 114 \\
\hline \multicolumn{5}{|l|}{ Edad (años) } \\
\hline$<15$ & 146 & 101 & 129 & 89 \\
\hline$\geq 15$ & 217 & 263 & 192 & 233 \\
\hline No responde & 5 & 4 & 4 & 3 \\
\hline
\end{tabular}


motivos relacionados con SSR. Esos pocos casos fueron pesquisados al revisar el motivo de consulta que declararon por el cuál habían solicitado atención. Poco más de la mitad de los estudiantes $\left(52,9 \% ; \mathrm{CI}_{95} 47,6-58,4\right)$ indicó estar informado de la existencia de atención de SSR en los servicios de atención primaria y de ellos, poco más de un quinto $\left(22,1 \% ; \mathrm{CI}_{95}\right.$ 15,9-28,2), había consultado. Entre quienes habían usado este tipo de servicios, la mayoría fueron mujeres $(81,7 \%)$, mostrando una diferencia por sexo estadísticamente significativa $(p<0,0001)$.

En el análisis bivariado, se identificaron 11 factores asociados con una probabilidad significativamente mayor de consultar los servicios de SRR respecto del grupo de referencia, los que se detallan en la tabla 2. La única variable que se asoció en dirección contraria al uso de los servicios de SSR fue contar con una actividad regular destinada al uso del tiempo libre. Sin embargo, en el modelo final multivariado sólo cuatro factores resultaron predictivos del uso de servicios de SSR, según se observa en la misma tabla 2 ya señalada: ser mujer; estar involucrado/a en una relación afectiva de pareja; haber recibido educación sexual en la familia; y no vivir con ninguno de sus padres. La distancia geográfica de la clínica no fue un elemento relevante, debido a que el $95 \%$ de los estudiantes vivía cerca de los servicios de atención primaria.

Los estudiantes que habían consultado por atención de SSR en los servicios públicos de atención primaria, evaluaron la atención recibida de manera positiva en su gran mayoría, más del $80 \%$ en todos los casos. No obstante, se observaron diferencias por edad como que los adolescentes mayores de 15 años se mostraron más satisfechos respecto a la solución del problema que generó la consulta, en comparación con los menores de 15 años: 93,4\% versus $50,0 \%$ respectivamente $(\mathrm{p}=0,016)$. El análisis por sexo no mostró diferencias significativas, aun cuando las mujeres tendieron a estar más conformes que los varones.

Las razones para no haber consultado por atención de SSR en los servicios públicos señaladas por los encuestados, se muestran en la tabla 3. Para las mujeres la vergüenza de consul- tar o el temor de ser vistas por alguien que les cuente a sus padres que las vieron consultando, fueron las razones más frecuentes, argumentos que arrojan diferencias estadísticamente significativas al ser comparados por sexo $(p=0,011$ y $\mathrm{p}=0,001$, respectivamente). Respecto de si los adolescentes consideran que no tienen derecho a utilizar los servicios de SSR, motivo casi inexistente para las mujeres y que fue indicado en un escaso porcentaje cercano sólo al 4\% de los adolescentes varones, marcó una diferencia estadísticamente significativa por sexo $(p<0,0001)$. El análisis por grupos de edad no mostró diferencias estadísticas.

Los no usuarios de servicios de SSR, también señalaron que el mejor lugar donde los adolescentes pueden buscar ayuda para resolver sus necesidades de salud en ésta área es el Consultorio de Atención Primaria del sistema público (tabla 4), lo que no muestra diferencias estadísticamente significativas ni por edad ni por sexo. Las mujeres consideraron como mejor alternativa para consultar, un Centro exclusivo de Adolescencia, en cambio para los varones fueron los Servicios de Urgencia. Dichos lugares mencionados arrojaron diferencias estadísticamente significativas por sexo en ambos casos. Aunque la farmacia fue seleccionada por menos del $10 \%$ de los estudiantes, esta alternativa es más prevalente en los menores de 15 años, siendo la diferencia por edad estadísticamente significativa.

El análisis de la pregunta sobre las recomendaciones para servicios amigables para adolescentes, que excluyó a quienes no respondieron y que consideró sólo el porcentaje de alumnos que marcó la mayor importancia (nivel 5) para cada una de las variables, arrojó diferencias estadísticamente significativas según sexo para las siguientes características: "Que pudiera ir solo(a)" (mujeres 50,5\%, hombres 25,3\%, p =0,0002); "Que no me diera vergüenza ir a consultar" (mujeres $72,2 \%$, hombres 51,6\%, $\mathrm{p}=0,0018$ ); "Que no me encontrara con adultos" (mujeres 46,3\%, hombres 31,6\%, $\mathrm{p}=0,027$ ); "Que no pidieran documentos que habitualmente están en poder de los padres (mujeres $68,2 \%$, hombres $45,3 \%$, $\mathrm{p}=0,0008) ; \mathrm{y}$ "Que no le contaran a la familia (mujeres $63,2 \%$, hombres $46,3 \%, \mathrm{p}=0,017$ ). 
Tabla 2. Factores asociados a la posibilidad de que los adolescentes consulten los servicios de SSR, Razón de Riesgo e Intervalo de Confianza al $\mathbf{9 5 \%}$ del análisis Bivariado y del Multivariado

\begin{tabular}{|c|c|c|c|c|c|c|}
\hline \multirow[t]{2}{*}{ Factores } & \multicolumn{3}{|c|}{ Bivariado } & \multicolumn{3}{|c|}{ Multivariado } \\
\hline & RD & $I_{95}$ & Valor p & RD & $I_{95}$ & Valor $\mathbf{p}$ \\
\hline \multicolumn{7}{|l|}{ Sexo } \\
\hline Mujer & 4,78 & $2,3-10,2$ & $<0,0001$ & 3,4 & $1,25-9,62$ & 0,017 \\
\hline Hombre & 1 & & & 1 & & \\
\hline \multicolumn{7}{|l|}{ Edad } \\
\hline$<15$ años & 1 & & & 1,56 & $0,63-3,81$ & NS \\
\hline$\geq 15$ años & 3,06 & $1,248-6,32$ & 0,0025 & 1 & & \\
\hline \multicolumn{7}{|l|}{ Fumar cigarrillos (tabaco) } \\
\hline Sí & 2,51 & $1,09-5,78$ & 0,031 & 2,10 & $0,77-5,7$ & NS \\
\hline No & 1 & & & 1 & & \\
\hline \multicolumn{7}{|c|}{ Consumo de sustancias (cannabis, alguna forma de cocaína) } \\
\hline Sí & 2,94 & $1,49-5,79$ & 0,0018 & 1,1 & $0,47-2,61$ & NS \\
\hline No & 1 & & & 1 & & \\
\hline \multicolumn{7}{|c|}{ Realizar regularmente actividades en el uso el tiempo libre } \\
\hline Sí & 0,34 & $0,12-0,74$ & 0,009 & 0,48 & $0,21-1,11$ & NS \\
\hline No & 1 & & & & & \\
\hline \multicolumn{7}{|c|}{ Percepción de autonomía para tomar sus propias decisiones } \\
\hline Sí & 2,63 & $1,38-5,01$ & 0,003 & 1,56 & $0,68-3,59$ & NS \\
\hline No & 1 & & & 1 & & \\
\hline \multicolumn{7}{|c|}{ Diferencia entre su edad actual y la mejor edad para iniciar actividad sexual } \\
\hline$\leq 1$ año & 3,55 & $1,84-6,86$ & 0,002 & 2,3 & $0,92-5,74$ & NS \\
\hline$>1$ año & 1 & & & & & \\
\hline \multicolumn{7}{|l|}{ Estructura familiar } \\
\hline Biparental & 1 & & & 1 & & \\
\hline Uniparental (madre o padre) & 1,38 & $0,68-2,8$ & NS & \multicolumn{2}{|c|}{ Excluida del modelo final } & \\
\hline Otros, sin los padres ${ }^{(1)}$ & 4,42 & $1,68-11,6$ & 0,0025 & 3,24 & $1,16-9,03$ & 0,024 \\
\hline \multicolumn{7}{|c|}{ Haber tenido educación sexual en la familia } \\
\hline Sí & 3,92 & $1,77-8,71$ & 0,0008 & 4,49 & $1,36-14,7$ & 0,013 \\
\hline No & 1 & & & 1 & & \\
\hline \multicolumn{7}{|c|}{ Estar inscrito en el Consultorio de su barrio u otro } \\
\hline Sí & 2,70 & $1,18-6,17$ & 0,018 & 1,9 & $0,65-5,57$ & NS \\
\hline No & 1 & & & & & \\
\hline \multicolumn{7}{|c|}{ Estar comprometido/a en una relación afectiva de pareja al momento de la encuesta (formal o informal) } \\
\hline Sí & 3,26 & $1,67-6,37$ & 0,005 & 2,6 & $1,03-6,78$ & 0,049 \\
\hline No & 1 & & & & & \\
\hline \multicolumn{7}{|c|}{ Haber tenido un embarazo o haber embarazado a una mujer } \\
\hline Sí & 5,01 & $1.97-12.7$ & 0,0007 & 2,14 & $0,8-5,7$ & NS \\
\hline No & 1 & & & & & \\
\hline \multicolumn{7}{|c|}{ Haber tenido la experiencia de un embarazo adolescente en la familia o entre los amigos } \\
\hline Sí & 2,07 & $1,05-4,08$ & 0,035 & Exclu & del modelo final & \\
\hline No & 1 & & & & & \\
\hline
\end{tabular}

(1) Vivir sin los padres: "Abuelos"; "Con la familia de mi pareja y mi hijo"; "Sobrina, cuñado y padre de mi hermana"; "Tíos, abuela y primos"; "Hermanos o hermanas"; "Padrastro". SSR: salud sexual reproductiva. 
Tabla 3. Razones por las cuales los adolescentes encuestados no habían consultado los Servicios Públicos de Salud Sexual y Reproductiva, según sexo

\begin{tabular}{|c|c|c|c|}
\hline Razones & $\begin{array}{l}\text { Mujeres } \\
(n=117)\end{array}$ & $\begin{array}{l}\text { Hombres } \\
(n=148)\end{array}$ & Valor $\mathbf{p}$ \\
\hline No he necesitado estos servicios & 61,4 & 57,2 & NS \\
\hline No creo que tenga derecho a usar esos servicios & 0,7 & 4,2 & $<0,0001$ \\
\hline No sabía que existían & 13,1 & 23,6 & NS \\
\hline Mis padres no estarían de acuerdo & 17,5 & 4,5 & NS \\
\hline No sé para qué sirven & 5,2 & 7,1 & NS \\
\hline Me da vergüenza consultar & 37,4 & 22,3 & 0,011 \\
\hline $\begin{array}{l}\text { Me preocupa que me pudiera encontrar con alguna persona que se lo cuente a } \\
\text { mis padres }\end{array}$ & 22,1 & 8,2 & 0,001 \\
\hline Otras & 17,1 & 3,0 & 0,017 \\
\hline
\end{tabular}

Tabla 4. Mejor lugar para consultar según adolescentes no consultantes de Servicios Públicos de Salud Sexual y Reproductiva, por sexo y edad

\begin{tabular}{|c|c|c|c|c|c|c|}
\hline \multirow[t]{2}{*}{ El mejor lugar para consultar } & \multicolumn{2}{|c|}{ Sexo $(\%)$} & \multirow[t]{2}{*}{ Valor $\mathbf{p}$} & \multicolumn{2}{|c|}{$\operatorname{Edad}^{(a)}(\%)$} & \multirow[t]{2}{*}{ Valor $p$} \\
\hline & $\begin{array}{c}\text { Mujer } \\
(n=117)\end{array}$ & $\begin{array}{l}\text { Hombre } \\
(n=148)\end{array}$ & & $\begin{array}{c}<15 \\
(n=81)\end{array}$ & $\begin{array}{l}15-18(+) \\
(n=181)\end{array}$ & \\
\hline En el consultorio (Sistema público) & 52,2 & 61,6 & NS & 50,8 & 57,6 & NS \\
\hline En una consulta particular (Sistema privado) & 22,3 & 29,6 & NS & 15,5 & 27,9 & NS \\
\hline En la farmacia & 6,1 & 5,7 & NS & 8,9 & 3,1 & 0,0122 \\
\hline En la posta o servicios de urgencia & 10,4 & 25,0 & 0,002 & 20,4 & 14,9 & NS \\
\hline En el colegio & 22,8 & 14,8 & NS & 8,9 & 19,8 & NS \\
\hline En un centro o unidad exclusiva para adolescentes & 71,1 & 30,6 & 0,0003 & 30,4 & 46,6 & NS \\
\hline En otros lugares: familia, amigos, etc. & 3,1 & 7,0 & NS & 3,5 & 5,1 & NS \\
\hline
\end{tabular}

(a)No responden: $\mathrm{n}=3$.

\section{Discusión}

Disminuir las barreras para que los y las adolescentes accedan a los servicios de SSR cuando los necesitan, es un tema del mayor interés para quienes formulan políticas y toman decisiones destinadas a facilitar la atención de salud a este grupo etario ${ }^{36,37}$. En este estudio, la principal razón declarada tanto por hombres como por mujeres adolescentes para no haber consultado por atención de SSR, fue no haberlo necesitado. Las características recomendadas para los servicios de SSR amigables para adolescentes parecen ser más importantes para las mujeres que para los hombres. Una barrera importante para ellas es, por una parte la vergüenza que les genera consultar a algún profe- sional del equipo de salud por atención de SSR $\mathrm{y}$, por otra parte, arriesgarse a ser vistas en el Consultorio de Atención Primaria por alguna persona que pueda informar a sus padres. Entre los hombres adolescentes no usuarios de servicios de SSR, prácticamente uno de cada cuatro dijo que no consultaba por desconocer la existencia de estos servicios. Es interesante observar una tendencia en los adolescentes hombres a conocer menos que las mujeres acerca de la atención de SSR y, en las mujeres, una mayor tendencia a pensar que sus padres no aprobarían que consultaran dichos servicios (tabla 3 ).

Como ya fuera mencionado, el estudio encontró cuatro variables predictivas del uso de servicios de SSR públicos: ser mujer, estar involucrado/a en una relación afectiva de 
pareja; haber recibido educación sexual en la familia y no vivir con ninguno de sus padres (tabla 2). Con relación a este último grupo, se podría plantear la hipótesis que en caso de no disponer de la presencia de la madre ni del padre en la vida cotidiana, los proveedores de salud resultan adultos referentes válidos para los adolescentes en temas sensibles como la sexualidad y la salud reproductiva ${ }^{33}$. La única variable asociada con el no uso de servicios de SSR fue tener alguna actividad regular, organizada, para el uso del tiempo libre.

Merece ser destacado que las evaluaciones de la atención de SSR recibida en los consultorios del sistema público de salud, resultaron mayoritariamente positivas. Casi dos tercios de los adolescentes consideraron la Atención Primaria del Sistema Público de Salud como el mejor lugar para recibir este tipo de atención, en especial las mujeres y los estudiantes mayores de 15 años. Sin embargo, para los adolescentes más jóvenes menores de 15 años, dichos servicios les resultaron menos resolutivos, aunque el hallazgo se basa en números pequeños. Justamente, es en este último grupo de edad en particular, en el que se concentran las consecuencias adversas de los embarazos a edades tempranas. Es posible observar que la proporción de nacidos vivos en adolescentes muy jóvenes se ha mantenido estable en Chile en a lo menos la última década, manteniéndose del orden de 1000 nacidos vivos anuales hijos de madres menores de 15 años, por lo que ningún esfuerzo dirigido a disminuir los nacimientos en este grupo específico ha dado, hasta ahora, los resultados esperados ${ }^{38}$.

Prácticamente todos los jóvenes estudiados, consideraron tener derecho a solicitar y recibir atención de SSR en los servicios públicos. Pocos estudiantes señalaron a las farmacias como un lugar válido para solucionar directamente sus necesidades en salud sexual y reproductiva. No hay que olvidar que el acceso a las farmacias está condicionado a la disponi- bilidad de recursos financieros y el grupo estudiado proviene de un sector socioeconómico en desventaja.

Como en otros países, los servicios de SSR en Chile tradicionalmente han estado dirigidos hacia las mujeres $\mathrm{y}$, en particular, a aquellas en edad reproductiva y con hijos, enfocándose en el tradicional concepto del cuidado de la salud Materno-Infantil. En este estudio, las mujeres solicitaban los servicios de SSR más frecuentemente que los hombres, así como también los adolescentes que se encontraban involucrados en una relación afectiva de pareja al momento del estudio, comparados con aquellos que no la tenían. De lo anterior se deduce que los consultantes, además de ser primordialmente mujeres, se encontraban más cerca de la probabilidad de iniciar o de haber iniciado actividad sexual y por lo tanto, de enfrentar el riesgo reproductivo. Los patrones de visitas a los servicios de SSR encontrados en este estudio, no difieren entonces de lo que se ha visto en el pasado en los servicios de salud Materno-Infantil.

La obtención del Consentimiento Informado de los padres para que sus hijos/as participaran en la encuesta, no estuvo exenta de dificultades como el retraso en responder y falta de retorno de muchos formularios, ni aceptando ni oponiéndose. Los Directores de los establecimientos educacionales, considerando el interés para sus alumnos y la pertinencia del tema a investigar, una vez revisado el instrumento para certificar que las preguntas y el lenguaje fuera adecuado al nivel de sus alumnos y, dado el carácter anónimo y confidencial de la encuesta, señalaron al equipo de investigación que ellos podrían otorgar la autorización escrita para que participaran los estudiantes cuyos padres no habían respondido. Este es un procedimiento legal en Chile, donde existe una disposición que autoriza a los Directores para tomar decisiones en materias de este tipo en su establecimiento educacional ${ }^{*, 39}$.

\footnotetext{
*Decreto del Ministerio de Educación y carta de autorización de los Directores: "El rol del Director es el de un profesional de alto nivel, basado en educación específica y experiencia requeridos para el cargo. Las responsabilidades del Director incluyen aspectos relativos a dirección, administración, supervisión y coordinación de la educación y comprenden tutoría directa y responsabilidad por el personal incluyendo aquellos profesores y no profesores, administrativo, auxiliar u otro personal y los estudiantes. Disponible en http://www.mineduc.cl/biblio/documento/DEC 453 1992.pdf
} 
La autorización de los directores para realizar la encuesta no eximió de manera alguna el proceso de Consentimiento Informado de los alumnos, condición previa si ne qua non para ser incluidos en el estudio.

\section{Limitaciones del estudio}

Los resultados de este estudio provienen de una muestra de adolescentes estudiantes de establecimientos educacionales públicos, del área urbana la Región Metropolitana de Chile, la que para el año 2005 concentraba el 44,8\% de la población del país ${ }^{40}$. Los hallazgos del estudio emanan de adolescentes estudiantes por lo que no pueden extenderse a aquellos que no asisten al sistema de educación formal, los que son una minoría. En Chile el 94,3\% de los niños entre 6 y 13 años y el 80,8\% de los adolescentes mayores de esa edad, asistían al colegio en el año $2007^{41}$. Es posible también que estos resultados no sean extrapolables a adolescentes de ciudades fuera del Área Metropolitana o de zonas rurales, donde vive sólo el $13,2 \%$ de la población ${ }^{38}$, ni a adolescentes de ciudades y/o comunidades con mayoría étnica de pueblos originarios.

\section{Conclusiones}

Sólo la mitad de los adolescentes cubiertos por el Sistema Público de Salud sabían que podían consultar por atención de SSR en los servicios de Atención Primaria y cerca de un quinto de esos beneficiarios había consultado al menos una vez.

La atención de SSR entregada en el sistema público de salud fue evaluada positivamente por los adolescentes estudiados. No obstante, sólo satisfacía parcialmente las necesidades de los adolescentes más jóvenes (menores de 15 años) y los que consultaron fueron mayoritariamente mujeres y aquellos que se encontraban en una relación afectiva de pareja. Por lo tanto, los consultantes por atención de SSR en los servicios públicos siguen respondiendo al clásico perfil de este tipo de servicios, dirigidos a mujeres y a personas sexualmente activas, por lo que habitualmente se marginan otro tipo de adolescentes que también la necesitan, en particular para recibir educación y prevención primaria.

Haber recibido educación sexual en la familia se asoció al uso de servicios de SSR, situación que podría resultar doblemente protectora. Por una parte, estos adolescentes tenían la percepción de haber recibido información y sido educados en sexualidad por sus padres $\mathrm{y}$, por otra parte, se relacionó con la búsqueda adecuada por parte de los y las adolescentes, de servicios especializados otorgados por profesionales capacitados, ya sea para requerir atención o consejería. Esto reviste importancia para países en que persiste un déficit de programas de educación sexual en los colegios, en especial en aquellos establecimientos que educan a la población más vulnerable, la que concentra los embarazos y que muchas veces no recibe educación sexual en la familia y, por otra parte, confirma que la educación sexual debe ir acompañada de la oferta de servicios.

Difundir información sobre la existencia de servicios de SSR parece muy necesario para mejorar el acceso de los y las adolescentes a éstos, destacando el rol de los profesionales en las actividades de educación, consejería y prevención. El enfoque preventivo y de orientación que realizan está destinado también a aquellos adolescentes que no han iniciado actividad sexual. Para desarrollar iniciativas de difusión efectiva, es importante tener en cuenta la recomendación de involucrar a los propios adolescentes en la elaboración de éstas.

Finalmente, un interesante desafío de investigación emerge de esta vivencia de trabajo de campo, como es conocer cuál es la percepción de padres de adolescentes vulnerables que cuentan con escasas herramientas de lecto-escritura y que no han sido educados en un contexto de derechos, respecto del proceso de consentimiento informado.

\section{Agradecimientos}

El equipo de investigadoras agradece a las comunidades de los establecimientos educacionales que colaboraron con esta investigación, especialmente a los respectivos Directores, y a los padres que autorizaron a sus hijos/as 
a participar en este estudio. Las autoras desean hacer llegar un reconocimiento especial a los y las estudiantes adolescentes que participaron voluntariamente en este estudio, cuya desinteresada y anónima participación contribuirá al conocimiento para mejorar los servicios públicos de salud sexual y reproductiva dirigidos a este grupo etario.

Desea también agradecer en forma especial, a Olav Meirik MD, Ph.D., por su importante contribución al proyecto y a la elaboración de esta publicación.

\section{Referencias}

1.- Blum R: Young people: not as healthy as they seem. The Lancet, 2009; 374: 853-4.

2.- WHO: The Adolescent Health \& Development (AHD) unit-Investing in a health sector response to Adolescents \& Young People. Available at: http://www.searo.who. int/EN/Section13/Section1245.htm. Accessed July 27, 2009.

3.- Luengo X: La Salud del Adolescente y Joven en Chile y los Servicios de Salud. Informe de Consultoría. Proyecto Apoyo a las Iniciativas Nacionales. OPS-OMS/ KELLOGG/MINSAL. Chile 1994.

4.- Patton G, Coffey $C$, Sawyer $S$, et al: Global patterns of mortality in young people: a systematic analysis of population health data. The Lancet 2009; 374: 881-92.

5.- McAnarney, Kreipe, Orr \& Comerci eds: Textbook of Adolescent Medicine. W.B. Saunders CompanyPhiladelphia P.A., 1992. ( $1^{\text {a }}$ edición en español, Buenos Aires-Argentina, Editorial Médica Panamericana, 1994).

6.- Dulanto E, ed: El Adolescente. McGraw-Hill Internacional. México D.F., 2000.

7.- Senderowitz J: Making Reproductive Health Services Youth Friendly (1997). Available at: http://www.fhi.org/ en/Youth/YouthNet/Publications/FOCUS/InFOCUS/ makingYFS.htm Accessed July 1, 2009.

8.- Recomendaciones para la Atención Integral de los y las Adolescentes con énfasis en salud sexual y reproductiva. OPS/OMS-UNFPA Serie $\mathrm{N}^{\circ}$ 2. Washington DC, 2000.

9.- Adolescent Friendly Health Services: An agenda for change. Geneva, Switzerland: World Health Organization, $\mathrm{WHO} / \mathrm{FCH} / \mathrm{CAH} / 02.14,2002$.

10.- IMAN Servicios: Normas de atención de salud sexual y reproductiva de adolescentes. Washington, DC: OPS/ OMS, 2005.
11.- Política Nacional de Salud para Adolescentes y Jóvenes. Santiago de Chile: Ministerio de Salud de Chile, División de Salud de las Personas, Programa de Salud del Adolescente, 1999.

12.- Política Nacional de Salud de Adolescentes y Jóvenes 2008-2015. Santiago de Chile: Gobierno de Chile, Ministerio de Salud, 2009.

13.- Luengo X, Tijero M, Zepeda A: El Programa de Salud de los y las Adolescentes. Chile 1995-2005. Análisis del estado de situación del programa dirigido a los y las adolescentes en el sistema de salud público de Chile. Santiago de Chile: UNFPA-ICMER Project Report, CHIOR201, 2006.

14.- Normas Nacionales sobre Regulación de la Fertilidad. Santiago de Chile: Ministerio de Salud, 2006.

15.- Sentencia ROL 740-07-CDS,18/04/2008: Santiago de Chile: Tribunal Constitucional. Disponible en: http:// www.anticoncepciondeemergencia.cl/legal.htm Visitado en diciembre 15, 2008.

16.- Society for Adolescent Medicine: Confidential Health Care for Adolescents: Position Paper. J Adolesc Health 2004; 35:1-8.

17.- Lehrer JA, Pantell R, Tebb K, Shafer MA: Forgone Health Care among U.S. Adolescents: Associations between Risk Characteristics and Confidentiality Concern. J Adolesc Health 2007; 40: 218-26.

18.- Churchill D, Allen J, Pringle M, et al: Consultation patterns and provision of contraception in general practice before teenage pregnancy: case-control study. BMJ, 2000; 321: 486-489.

19.- Discussion paper on Adolescence: Adolescent Pregnancy. Issues on Adolescent Health and Development. Geneva: World Health Organization, Department of Child and Adolescent Health, Department of Reproductive Health and Research, 2004.

20.- Giving Girls Today \& Tomorrow: Breaking the Cycle of Adolescent Pregnancy. New York: UNFPA, 2007.

21.- Boonstra HD: Young People Need Help in Preventing Pregnancy and HIV; How Will the World Respond? Guttmacher Policy Review 2007; 10: 2-8.

22.- Perfil de salud sexual y reproductiva de los y las adolescentes y jóvenes de América Latina y El Caribe: revisión bibliográfica, 1988-1998. Washington DC: OPS/ FNUAP Serie $N^{\circ} 1,2000$.

23.- Schutt-Aine J, Maddaleno M: Sexual Health and Development of Adolescents and Youth in the Americas: Program and Policy implications. Washington DC : PAHO, Adolescent Health and Development Unit, Family Health and Population Program, Division of Health Promotion and Protection, HIV/AIDS Program, 
Division of Disease Prevention and Control, 2003.

24.- Luengo X: Necesidades de salud. En Molina R, Sandoval J y González E, eds. Salud Sexual y Reproductiva en la Adolescencia. Santiago de Chile, Editorial Mediterráneo, 2003: 102-10.

25.- Los Objetivos del desarrollo del Milenio: Objetivo 5. Indicadores adicionales ODM. Meta $\mathrm{N}^{\circ}$ 2. Disponible en: http://siis.mideplan.cl/indicadoresmilenio/ Visitado en marzo 31, 2009.

26.- World Health Statistics 2008: World Health Organization. Geneva, 2008.

27.- State of World Population 2008: Reaching Common Ground: Culture, Gender and Human Rights. UNFPA, 2008.

28.- Gobierno de Chile, Instituto Nacional de la Juventud (INJUV): Prácticas y Estilos de Vida de los y las Jóvenes del siglo XXI. Estudios del INJUV, Vol 3. Santiago, Chile 2005.

29.- Dides C, Morán JM, Benavente R, Pérez S: Salud Sexual y Reproductiva en Chile 2007: actualización de datos estadísticos. Programa de Género y Equidad. Santiago de Chile, UNFPA/FLACSO, 2008.

30.- Instituto Nacional de la Juventud, Gobierno de Chile: Sexta Encuesta Nacional de Juventud: Capítulo Sexualidad, Análisis Preliminares. Chile, 2009.

31.- Orientaciones para la implementación de Servicios de Salud Amigables para Adolescentes y Jóvenes en ChileDocumento final. UNFPA, Proyecto CHI1R05. Ministerio de Salud, Programa Nacional de Adolescentes y Jóvenes en Chile. Enero 2008

32.- Resultados del estudio educación en sexualidad. Gobierno de Chile, Ministerio de Salud y Adimark. Chile,
2004.

33.- Sánchez-Crespo JL: Curso Intensivo de Muestreo en Poblaciones Finitas. Instituido Nacional de Estadísticas (INE). Madrid. España. 1980.

34.- Gatsonis C, Sampson AR: "Multiple Correlation: Exact Power and Sample Size Calculations" Psychological Bulletin 106(1989) pp. 516-24.

35.- SAS Institute Inc., SAS/STAT Software (9.1 Version), Cary, NC: SAS Institute Inc., 2006.

36.- Society for Adolescent Medicine: Acces to Health Care for Adolescents and Young Adult: Position Paper. J Adolesc Health 2004; 35: 342-4.

37.- Tylee A, Haller DM, Graham T, Churchill R, et al: Youth-friendly primary-care services: how are we doing and what more needs to be done? Adolescent Health 6 . Lancet 2007; 369: 1565-73.

38.- Molina CR, Molina GT, González E: "Madres niñasadolescentes de 14 años y menos. Un grave problema de salud pública no resuelto en Chile". Rev Med Chile 2007; 135: 79-86.

39.- Ministerio de Educación (MINEDUC): Estatuto de los Profesionales de Educación. Disponible en: http://www. mineduc.cl/biblio/documento/DEC_453_1992.pdf

40.- Estadísticas Vitales: Informe Anual 2005. Gobierno de Chile. Instituto Nacional de Estadísticas. Marzo 2008.

41.- Indicadores de la educación en Chile 2007: Documento Preliminar. Gobierno de Chile, Ministerio de educación. Departamento de Estudios y Desarrollo, División de Planificación y Presupuesto. Disponible en: http:// w3app.mineduc.cl/mineduc/ded/documentos/Indicadores_de_la_Educacion_2007.pdf (Visitado en agosto 24, 2009) 